\title{
Partial caries removal and cariostatic materials
}

\author{
Partial caries removal and cariostatic materials in carious primary molar teeth: a randomised controlled clinical trial \\ J. Foley, D. Evans and A. Blackwell Br Dent J 2004; 197:697-701
}

\section{Objective}

To determine the durability and effectiveness of a black copper cement (BCC) and a conventional glass ionomer cement (GIC) when used to restore primary molars following partial caries removal (PCR) and to compare these results with conventional cavity preparation and restoration.

\section{Design and Setting}

Split-mouth randomised controlled clinical trial. Department of Paediatric Dentistry, Dundee Dental Hospital, Dundee, 1998-1999.

\section{Subjects}

Patients with previously unrestored, matched carious cavities in non-pulpally involved primary molars.

\section{Interventions}

Three treatment groups: (1) Partial caries removal followed by lining with BCC and restoration with GIC (PCR:BCC); (2) Partial caries removal and restoration with GIC alone (PCR:GIC), and (3) Complete caries removal and conventional restoration (CR). Restoration durability and effectiveness was assessed both clinically and radiographically over 24 months.

\section{Main outcome measures}

Median survival time (MST) of restorations.

\section{Results}

Forty-four patients (F: 31; M: 13), mean age 6.8 years (range: 3.7-9.5), had 120 restorations placed (PCR:GIC: 43; CR: 41; PCR:BCC: 36). Eighty-six molars (29 patients) (PCR:GIC: 30; CR: 29; PCR:BCC: 27) were reviewed at 24 months. The median survival times (MST) with 25\% and 75\% quartiles in parenthesis were as follows: PCR:BCC, MST $=24$ months $(6,24)$; PCR:GIC, MST $=24$ months $(24$, $24)$ and $C R, M S T=24$ months $(24,24)$. The MST for PCR:BCC restorations was significantly less than for PCR:GIC and CR restorations ( $\mathrm{W}=1163.5, P=0.028$ and $\mathrm{W}=1081.0, P=0.004$ respectively).

\section{Conclusion}

There were no differences in the proportions of restorations lost between restoration types, although PCR:BCC restorations did have significantly more abscess/sinus formation over the 24month study period.

\section{IN BRIEF}

- Partial caries removal in primary teeth, lining with BCC and restoration with GIC showed poor durability over a 2-year period.

- Such restorations, however, demonstrated no significant progression in the carious process.

\section{COMMENT}

A treatment of carious lesions in primary molar teeth that might be more acceptable to GDPs is explored in this paper. The study found similar failure rates whilst comparing partial caries removal and placement of either glass ionomer cement (GIC) or black copper cement (BCC) with complete caries removal and restoration with GIC.

In considering the findings, around $30 \%$ of the restorations were lost to follow-up after 24 months; eight due to abscess formation and two due to exfoliation. Of the remaining 86 , a further 31 (36\%) failed either because of active caries (29) or loss of restoration, nine of the teeth demonstrated an abscess, three had pulp therapy/extra coronal restoration carried out and six were extracted. The failures occurred within the first six months of placement and $62 \%$ were Class II. It is noted that restorations placed in second primary molars had a better survival than first primary molars, as indeed did Class I compared with Class II. The MST for all three categories was similar and censored at 24 months. The authors attributed the poor performance of the PCR: BCC restoration to BCC's solubility and undermining the GIC leading to failure.

Previous studies have explored different materials and survival rates as a means to restore the primary dentition, most of which demonstrated good survival rates but associated with local analgesia, isolation techniques and following basic principles of caries removal and cavity design. ${ }^{1,2}$ In an ideal world, removal of caries, pulp therapy where necessary and restoration using the most appropriate material would be acceptable for all children. However, this is not always possible, especially in relation to the child's age and co-operation. Therefore procedures to overcome these difficulties might be welcomed, especially if the number of children receiving some sort of restorative care in the primary dentition increased. The longevity of restorations has been discussed, and the authors consider whether or not efforts should be made to restore the primary dentition which is said to have a maximum normal life of 8-9 years. In the primary dentition the stainless steel crown is the best restoration, especially when more than two surfaces are involved. If BCC is considered, it is inevitable that when placed in unprepared cavities, it is more likely to fail because it is highly soluble. So not surprisingly in the PCR: BCC group the failure rate was much higher.

The questions that arise from this study are complex and will inevitably lead to further debate as to how the primary dentition should be treated if at all. The BSPD in its clinical guidelines ${ }^{3}$ is clear that all children should be afforded the best possible treatment and that the primary dentition should be restored. However, recent publications would suggest that it may not be necessary to fully restore the primary dentition as children are no better off by doing so. ${ }^{4}$

E. S. Davenport, Professor of Dental Education, Barts and the London, Queen Mary's School of Medicine and Dentistry doi:10.1038/sj.bdj.4811864

1. Mass E, Gordan M, Fuks A B. Assessment of compomer proximal restorations in primary molars: A retrospective study. J Dent Child 1999; 66: 93-97.

2. Fuks A B, Araujo FB, Osorio L B, et al. Clinical and radiographic assessment of Class II esthetic restorations in primary molars. Paediatr Dent 2000; 22: 479-485.

3. British Society of Paediatric Dentistry. A Policy document on management of caries in the primary dentition. Int J Paed 2001; 11: 153-157.

4. Tickle $M$, Milsom K, King D, et al. Carious primary teeth: their fate in your hands. $\mathrm{Br}$ Dent J2002; 192: 219-233. 